\title{
Pharmacological approach to the treatment of acromegaly
}

\author{
SHEREEN EzZAT, M.D., F.R.C.P.(C) \\ Division of Endocrinology and Metabolism, University of Toronto, Ontario, Canada
}

\begin{abstract}
The treatment of patients with persistently active acromegaly has been facilitated over the past decade by the advent of highly specific and selective pharmacological agents. Somatostatin analogs, derived from the native inhibitory hormone somatostatin, are available in extended-duration preparations and are effective in reducing serum levels of growth hormone (GH) and insulin-like growth factor-I (IGF-I) as well as in improving the adverse clinical effects of acromegaly. Cabergoline, an agonist with a specificity for the dopamine D-2 receptor, has been shown to suppress IGF-I levels and induce tumor shrinkage in 35 and $50 \%$ of patients, respectively. The GH receptor antagonists compete with naturally occurring $\mathrm{GH}$ for binding with the $\mathrm{GH}$ receptor. As such, pegvisomant normalizes circulating IGF-I levels in 80 to $90 \%$ of patients with acromegaly. This last line of therapy should be considered for use in patients in whom surgery and medical therapy with somatostatin and/or dopamine agonists are either ineffective or poorly tolerated.
\end{abstract}

\section{KEY WORDS - acromegaly - growth hormone • octreotide • lanreotide • cabergoline $\bullet$ pegvisomant}

The last decade has provided major progress in the development of highly specific and selective pharmacological agents that have greatly facilitated a more aggressive approach to the treatment of patients with persistently active acromegaly. In this article we will summarize current evidence for each of the major classes of pharmacotherapeutic agents, with special emphasis on their recognized benefits and risks. An attempt will be made to highlight patient subpopulations for whom unique combinations of treatment options should be considered.

\section{PHARMACOTHERAPEUTIC AGENTS}

\section{Somatostatin Analogs}

Derived from the native inhibitory hormone somatostatin, this class of agents represents a physiologically based approach to treating GH excess. The first-generation analogs had a halflife that lasted 2 hours longer than that of the native hormone and required subcutaneous administration at least three times a day. This class of analogs successfully reduces GH and IGF-I levels in 50 to $70 \%$ of patients and provides an IGF-I normalization rate of approximately $30 \%$ in patients in whom pituitary surgery has failed. ${ }^{11}$ Maximal suppression of hormone is reached within 2 hours and usually lasts for 6 hours. Because the decline in the GH level is rapid, the assessment of suppression can be made at the outset to identify the patient's potential responsiveness to octreotide. In patients in whom GH levels return to baseline before the end of the

Abbreviations used in this paper: $\mathrm{CDN}=$ Canadian; $\mathrm{GH}=$ growth factor; IGF-I = insulin-like growth factor-I; LAR = long-acting release. dosing interval, the frequency of administration can be further increased. Increasing the daily dose beyond 300 to $600 \mu \mathrm{g} /$ day rarely achieves a greater effect. ${ }^{9}$ To address some of these shortcomings, newer formulations of somatostatin analogs (octreotide-LAR and lanreotide-sustained release) with more extended durations of action have been developed. Administered in single intramuscular injections (10-40 mg every 28 days), these analogs result in similar, if not greater efficacy than that achieved with the subcutaneous formulations. ${ }^{2}$ The effects are more sustained than those of subcutaneous preparations and, therefore, compliance is presumably enhanced. Somatostatin analog responsiveness has been correlated with the somatostatin receptor subtype in some, ${ }^{18}$ but not all studies. ${ }^{4,8}$ The GH inhibitory effects of octreotide are also significantly better in patients who harbor densely granulated somatotroph adenomas compared with those with sparsely granulated adenomas. ${ }^{10}$ Sparsely granulated GH adenomas should be suspected in patients who present at an older age with a minimal elevation $(1-5 \mathrm{ng} / \mathrm{ml})$ of nonsuppressible GH levels. The main adverse events associated with the use of a somatostatin analog include transient abdominal cramps and malabsorptive diarrhea. There is an increased incidence of gall bladder sludge and stone formation, but these are not typically of clinical significance. ${ }^{17}$ These long-acting somatostatin preparations are expensive ${ }^{22}$ and require a meticulous reconstitution technique immediately before injection, compared with subcutaneous preparations. This renders a dedicated physician-nurse care-provider team essential for long-term care. It is anticipated that newer somatostatin receptorselective analogs will prove to be even more effective ${ }^{19}$ and easier to administer than currently available agents. 
Impact of Therapy On Disease Outcomes. It is relatively recently that a consensus has started to emerge on the definition of a safe or acceptable degree of acromegaly control. ${ }^{12}$ As such, the prospective examination of the impact of medical management on the rate of acromegalyassociated mortality has just started. Nevertheless, the results of retrospective studies support the role of adjunctive medical therapy in normalizing the level of IGF-I and mortality rates in patients with postoperative persistent disease..$^{20}$

The impact of somatostatin analogs on acromegaly related comorbid complications has been best studied in impaired cardiovascular function. Glucose-suppressed $\mathrm{GH}$ levels lower than $1 \mathrm{ng} / \mathrm{ml}$ and age-normalized IGF-I levels are associated with a significant improvement in cardiac function. ${ }^{3}$ In a multicenter prospective study, lantreotide therapy was associated with improvement in left ventricular hypertrophy and arrhythmias in patients with acromegaly. ${ }^{15}$ In observational studies involving patients with persistently active disease, octreotide treatment was shown to reduce prostate size and volume ${ }^{5}$ and to improve joint pain and active and passive articular mobility, while reducing joint thickness. ${ }^{6}$ Similarly, several indices of the severity of sleep apnea improve favorably in response to long-term ( $\geq 6$-month) octreotide-LAR treatment. ${ }^{13}$ Glucose intolerance or diabetes can be favorably or more rarely, detrimentally influenced by somatostatin analogs, ${ }^{14}$ emphasizing the need for longitudinal monitoring of glycemic control. Long-term somatostatin analog treatment has been associated with a significant reduction in insulin, triglyceride, and fibrinogen levels and improved intimal thickening in the carotid artery. ${ }^{16}$ The impact of medical therapy on concrete cardiovascular end points, including myocardial ischemia or infarction, remains to be proven.

Somatostatin analogs alleviate many symptoms related to acromegaly including headaches, sweating, and arthralgias in nearly $75 \%$ of patients. ${ }^{11}$ A reduction in the pituitary tumor size, however, is limited to a smaller subset of patients. The frequency and extent of tumor shrinkage appears to be greater ( $70 \%$ and $30 \%$, respectively) in patients who have not received previous therapy, ${ }^{2}$ compared with those who have tumor remnants following pituitary surgery ( $25 \%$ of patients). ${ }^{11}$

\section{Dopamine Agonists}

Traditionally, nonselective dopamine agonists have been considered to be ineffective in adequately controlling acromegaly disease activity. In more recent studies, however, the authors have asserted that cabergoline, which has been shown to be a relatively more selective agonist of the dopamine D-2 receptor, may provide greater benefit than formerly used dopaminergic agents. In dose-adjusted studies, cabergoline was shown to suppress IGF-I below $300 \mathrm{ng} / \mathrm{ml}$ (the approximate normal value for a patient $30-40$ years of age) in approximately $35 \%$ of patients depending on baseline IGF-I levels. As previously recognized, the concomitant excess of prolactin in patients with acromegaly is predictive of a better response to dopamine agonists, with an IGF-I level below $300 \mathrm{ng} / \mathrm{ml}$ attained in nearly $50 \%$ of such patients. ${ }^{1,7} \mathrm{~A}$ significant degree of tumor shrinkage was demonstrated in a surprising 50\% of patients. The addition of cabergoline to lanreotide in a subset of surgery- and octreotide-resistant cases results in significantly greater GH and IGF-I suppression than that attained using lanreotide alone. ${ }^{16}$ Adverse effects include abdominal cramps and orthostatic hypotension. The results of these limited and uncontrolled studies indicate that the use of cabergoline for acromegaly may be worthy of consideration in the patient with treatment-resistant acromegaly. It should be considered as an adjunct in patients with persistently active disease, particularly when their disease is associated with a concomitant excess of prolactin.

\section{Growth Hormone Receptor Antagonists}

The GH-receptor antagonists represent a relatively new class of therapy. The currently available agent was developed to compete with naturally occurring $\mathrm{GH}$ for binding with the $\mathrm{GH}$ receptor. Unlike native $\mathrm{GH}$, however, this $\mathrm{GH}$ antagonist prevents the dimerization and signaling of the GH receptor, resulting in reduced production of IGFI. Clinical trials have demonstrated that daily subcutaneous administration (10-20 mg) of pegvisomant results not only in reduction but in normalization of circulating IGFI levels in nearly 80 to $90 \%$ of patients with acromegaly. ${ }^{21}$ This agent, which recently was approved by the US Food and Drug Administration, appears to be well tolerated and does not produce any recognized significant adverse effects or tachyphylaxis after 1 year of continuous use. ${ }^{21}$ The long-term safety and impact on pituitary tumor or tumor remnant growth is currently under investigation. At this time, pegvisomant should be considered for use in patients in whom surgery and medical therapy with somatostatin and/or dopamine agonists have proved ineffective or poorly tolerated (Fig. 1).

\section{SELECTION OF THE APPROPRIATE AGENT}

The influence of disease control on comorbid conditions has supported the use of medical therapy in patients who have undergone pituitary surgery and who continue to have elevated GH and IGF-I levels. In addition, medical therapy has been advocated for subsets of patients with acromegaly including those who refuse or cannot gain access to an experienced pituitary neurosurgeon, those who are poor candidates for surgery or anesthesia and those in whom no discrete pituitary lesion can be identified. For those with large invasive lesions that clearly cannot be completely resected, medical therapy should initially be advocated for at least 3 to 6 months. Depending on outcome, surgery may then be reconsidered (Fig. 1).

\section{Cost Considerations}

Acromegaly is a disease that is accompanied by a significant economic burden. Longitudinal assessment of the economic costs relative to clinical and biochemical outcomes was examined over a 4-year period in 53 Canadian patients. ${ }^{22}$ The mean annual cost per patient was CDN \$8111 (95\% confidence interval CDN \$5848-\$10,374). The GH-lowering medications constituted the highest component (nearly 38\%) of the overall cost of disease management. It should be emphasized that, although surgical costs per patient were high (CDN \$2800-\$9200), the 


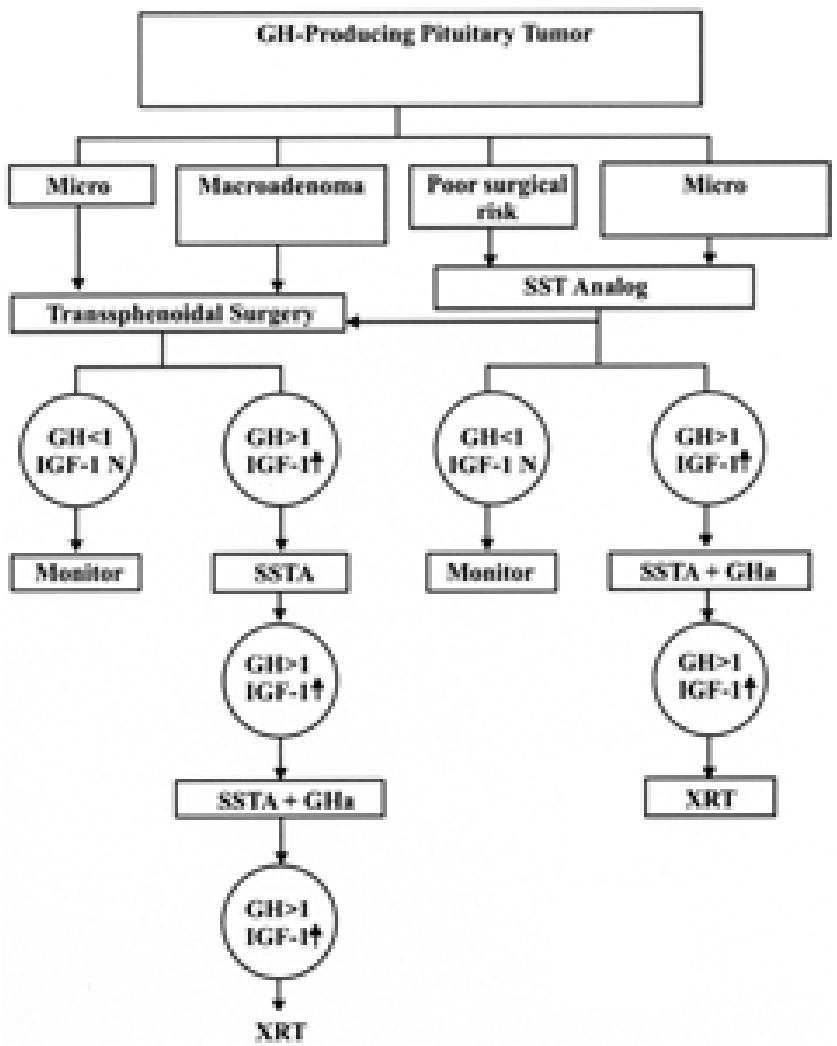

Fig. 1. Treatment algorithm for acromegaly. The GH levels (given in $\mathrm{ng} / \mathrm{ml}$ ) represent postoperative values obtained after an oral glucose tolerance test or a random value obtained after 3 months of sandostatin-LAR treatment. Insulin-like growth factor-I levels should be standardized for age- and sex-specific differences. Cabergoline may be added to other pharmacological therapies at any stage if the levels of GH and IGF-I are not normalized. Short arrow pointing upward indicates elevated; $\mathrm{GHa}=$ growth hormone antagonist; Micro = microadenoma; $\mathrm{N}=$ normal; SSTA = somatostatin analog therapy; XRT = radiotherapy.

4-year mean annual cost of surgery was approximately CDN \$2400 less than the cost of medications. Furthermore, treatment of patients with macroadenomas cost considerably more (CDN $\$ 11,425)$ than that of patients with microadenomas (CDN $\$ 4442$ annual cost). Although these are considerable costs, they are not significantly higher than those associated with other chronic diseases. ${ }^{22}$

\section{CONCLUSIONS}

Acromegaly is often a chronic debilitating condition that, if left uncontrolled, is associated with increased rates of morbidity and mortality. The diagnosis is established by documenting autonomous GH hypersecretion and by imaging of the pituitary gland. Resection of the responsible pituitary adenoma has traditionally represented the cornerstone of disease management. This has recently been challenged, however, because a strict target of agenormalized IGF-I and a glucose-suppressed GH level lower than $1 \mathrm{ng} / \mathrm{ml}$ is difficult to achieve using surgery alone. Adjunctive therapy is frequently necessary because complete resection is not always achievable. This has prompted the view that primary medical therapy may be a suitable option to consider for some patients. Persistently active disease is documented to be associated with increased risks of morbidity and mortality. A suggested therapeutic algorithm is shown in Fig. 1. Somatostatin analogs are of particular benefit to those patients with persistently nonsuppressible levels of $\mathrm{GH}$ and/or elevated IGF-I levels after pituitary surgery or during the interim period following radiotherapy. They may also constitute primary therapy for those patients who decline surgery, are not likely to obtain a remission of their disease, or cannot tolerate surgery or radiation treatment. The use of GH antagonists alone or in combination with somatostatin analogs, although rational from the pharmacological point of view, remains to be proven.

\section{References}

1. Abs R, Verhelst J, Maiter D, et al: Cabergoline in the treatment of acromegaly: a study in 64 patients. J Clin Endocrinol Metab 83:374-378, 1998

2. Bevan JS, Atkin SL, Atkinson AB, et al: Primary medical therapy for acromegaly: an open, prospective, multicenter study of the effects of subcutaneous and intramuscular slow-release octreotide on growth hormone, insulin-like growth factor-I, and tumor size. J Clin Endocrinol Metab 87:4554-4563, 2002

3. Clayton RN: Cardiovascular function in acromegaly. Endocr Rev 24:272-277, 2003

4. Colao A, Marzullo P, Lombardi G, et al: Effect of a six-month treatment with lanreotide on cardiovascular risk factors and arterial intima-media thickness in patients with acromegaly. J Clin Endocrinol 146:303-309, 2002

5. Colao A, Marzullo P, Spiezia S, et al: Effect of two years of growth hormone and insulin-like growth factor-I suppression on prostate disease in acromegalic patients. J Clin Endocrinol Metab 85:3754-3761, 2000

6. Colao A, Marzullo P, Vallone G, et al: Ultrasonographic evidence of joint thickening reversibility in acromegalic patients treated with lanreotide for 12 months. Clin Endocrinol 51: 611-618, 1999

7. Cozzi R, Attanasio R, Barausse M, et al: Cabergoline in acromegaly: a renewed role for dopamine agonist treatment? Eur J Endocrinol 139:516-521, 1998

8. Danila DC, Haidar JN, Zhang X, et al: Somatostatin receptorspecific analogs: effects on cell proliferation and growth hormone secretion in human somatotroph tumors. J Clin Endocrinol Metab 86:2976-2981, 2001

9. Ezzat S, Forster MJ, Berchtold P, et al: Acromegaly. Clinical and biochemical features in 500 patients. Medicine 73: 233-240, 1994

10. Ezzat S, Kontogeorgos G, Redelmeier DA, et al: In vivo responsiveness of morphological variants of growth hormone-producing pituitary adenomas to octreotide. Eur J Endocrinol 133: 686-690, 1995

11. Ezzat S, Snyder PJ, Young WF, et al: Octreotide treatment of acromegaly. A randomized, multicenter study. Ann Intern Med 117:711-718, 1992

12. Giustina A, Barkan A, Casanueva FF, et al: Criteria for cure of acromegaly: a consensus statement. J Clin Endocrinol Metab 85:526-529, 2000

13. Ip MS, Tan KC, Peh WC, et al: Effect of Sandostatin LAR on sleep apnoea in acromegaly: correlation with computerized tomographic cephalometry and hormonal activity. Clin Endocrinol 55:477-483, 2001

14. Koop BL, Harris AG, Ezzat S: Effect of octreotide on glucose tolerance in acromegaly. Eur J Endocrinol 130:581-586, 1994 
15. Lombardi G, Colao A, Marzullo P, et al: Improvement of left ventricular hypertrophy and arrhythmias after lanreotide-induced GH and IGF-I decrease in acromegaly. A prospective multi-center study. J Endocrinol Invest 25:971-976, 2002

16. Marzullo P, Ferone D, Di Somma C, et al: Efficacy of combined treatment with lanreotide and cabergoline in selected therapyresistant acromegalic patients. Pituitary 1:115-120, 1999

17. Newman CB, Melmed S, Snyder PJ, et al: Safety and efficacy of long-term octreotide therapy of acromegaly: results of a multicenter trial in 103 patients - a clinical research center study. J Clin Endocrinol Metab 80:2768-2775, 1995

18. Reubi JC, Landolt AM: The growth hormone responses to octreotide in acromegaly correlate with adenoma somatostatin receptor status. J Clin Endocrinol Metab 68:844-850, 1989

19. Saveanu A, Gunz G, Dufour H, et al: Bim-23244, a somatostatin receptor subtype 2- and 5-selective analog with enhanced efficacy in suppressing growth hormone $(\mathrm{GH})$ from octreotideresistant human GH-secreting adenomas. J Clin Endocrinol Metab 86:140-145, 2001
20. Swearingen B, Barker FG II, Katznelson L, et al: Long-term mortality after transsphenoidal surgery and adjunctive therapy for acromegaly. J Clin Endocrinol Metab 83:3419-3426, 1998

21. van der Lely AJ, Hutson RK, Trainer PJ, et al: Long-term treatment of acromegaly with pegvisomant, a growth hormone receptor antagonist. Lancet 358:1754-1759, 2001

22. Wilson LS, Shin JL, Ezzat S: Longitudinal assessment of economic burden and clinical outcomes in acromegaly. Endocr Pract 7:170-180, 2001

Manuscript received February 26, 2004.

Accepted in final form March 26, 2004.

Address reprint requests to: Shereen Ezzat, M.D., F.R.C.P.(C), Mount Sinai Hospital, University of Toronto, 600 University Avenue, Room 437, Toronto, Ontario M5G 1X5 Canada. email: sezzat @ mtsinai.on.ca. 